\title{
Increased serum IL-6 is predictive of long-term cardiovascular events in high-risk patients submitted to coronary angiography: an observational study.
}

\section{Márcio Mossmann}

Hospital de Clinicas de Porto Alegre https://orcid.org/0000-0002-7516-3416

Marco Vugman Wainstein MD

Hospital de Clinicas de Porto Alegre

Stéfani Mariani

Hospital de Clinicas de Porto Alegre

Guilherme Pinheiro Machado MD

Hospital de Clinicas de Porto Alegre

Gustavo Neves Araujo

Hospital de Clinicas de Porto Alegre

Sandro Cadaval Gonçalves MD

Hospital de Clinicas de Porto Alegre

Marcello Bertoluci ( $\square$ mcbertoluci@gmail.com )

Original investigation

Keywords: Interleukin-6, coronary artery disease, diabetes, high-sensitive C-reactive protein, inflammation

Posted Date: February 23rd, 2021

DOI: https://doi.org/10.21203/rs.3.rs-198311/v1

License: (c) (1) This work is licensed under a Creative Commons Attribution 4.0 International License.

Read Full License 
1 Increased serum IL-6 is predictive of long-term cardiovascular events in high-risk

2 patients submitted to coronary angiography: an observational study.

3

4 Márcio Mossmann MD, MSc'1; Marco Vugman Wainstein MD, $\mathrm{PhD}^{\mathbf{1 , 2}, 4}$; Stéfani Mariani

5 MD; Guilherme Pinheiro Machado MD, MSc ${ }^{1}$; Gustavo Neves de Araújo MD, $\mathrm{PhD}^{1}$;

6 Sandro Cadaval Gonçalves $\mathrm{MD}, \mathrm{PhD}^{1,2}$; Marcello Bertoluci $\mathrm{MD}, \mathrm{PhD}^{3,4}$

7

8 1- Universidade Federal do Rio Grande do Sul, Post-Graduate Program in Medical

9 Sciences: Cardiology and Cardiovascular Sciences, Porto Alegre, Brazil

10 2- Cardiology Division, Hospital de Clínicas de Porto Alegre, Porto Alegre, Brazil

11 3- Endocrinology Division, Hospital de Clínicas de Porto Alegre (HCPA), Universidade

12 Federal do Rio Grande do Sul (UFRGS), Porto Alegre, Rio Grande do Sul, Brazil.

4- Internal Medicine Department, School of Medicine, UFRGS, Porto Alegre, Rio Grande do Sul, Brazil.

Corresponding author:

Marcello Casaccia Bertoluci

Endocrinology Unit - Hospital de Clínicas de Porto Alegre - Ramiro Barcelos 2350, 90035-003.

Porto Alegre, RS, Brazil Phone: +55-51-98211.9898; Fax: +55-51-3359.8127 
ABSTRACT

BACKGROUND: Interleukin-6 (IL-6) is an inflammation-related cytokine associated

with an elevated risk of cardiovascular events. In a previous study, we demonstrated that increased IL-6 was predictive of sub-clinical atherosclerotic coronary disease in intermediate-risk patients undergoing coronary angiography. In the present study, we investigated whether increased serum IL-6 is predictive of cardiovascular events in highrisk patients.

METHODS: In this observational study, consecutive patients referred for elective coronary angiography due to stable chest pain/myocardial ischemia had IL-6 measured immediately before the procedure. Long-term follow-up was performed by phone call or e-mail, and their clinical registries were revised. The primary outcome was a composite of new myocardial infarction, new ischemic stroke, hospitalization due to heart failure, new coronary revascularization, cardiovascular death, and death due to all causes.

RESULTS: From the 141 selected patients, 100 completed the IL- 6 analysis and were followed for a mean of 5.5 years. The median age was 61.1 years, $44 \%$ were men, and $61 \%$ had type- 2 diabetes. The median overall time-to-event for the primary outcome was 297 weeks $(95 \%$ confidence interval $[\mathrm{CI}]=266.95-327.16)$. A receiver operator characteristic curve defined the best cut-off value of baseline serum IL-6 $(0.44 \mathrm{pg} / \mathrm{mL})$ with sensitivity (84.37\%) and specificity (38.24\%) to define two groups. High IL-6 level was moderately predictive of cardiovascular events. $(p$ for interaction $=0.015$ ) (hazard ratio $=2.81 ; 95 \% \mathrm{CI}=1.38-5.72, \mathrm{p}=0.01)$. The subgroup analysis did not find interactions between patients with or without diabetes, obesity, or hypertension.

CONCLUSION: This preliminary study indicates that, in high-risk symptomatic patients undergoing elective coronary angiography, increased pre-procedure serum levels of IL-6 predicted long-term cardiovascular outcomes. These results were similar irrespective of 
diabetes, hypertension, or obesity status. IL-6 must be studied in larger long-term followup studies as a potential tool to re-classify patients with increased cardiovascular risk.

Keywords: Interleukin-6, coronary artery disease, diabetes, high-sensitive C-reactive protein, inflammation.

\section{BACKGROUND}

Interleukin-6 (IL-6) is an acute-phase protein that plays a significant role in the inflammatory response, vascular inflammation, and atherosclerosis process [1]. It contributes to remodeling of connective tissue by increasing metalloproteinase gene expression [2]. Focal overexpression of activated metalloproteinase may promote destabilization and degradation of the plaque's fibrous cap, leading to plaque instability during the atherosclerotic process [3]. In a study including patients with unstable coronary artery disease (CAD), higher IL-6 levels $(>5 \mathrm{pg} / \mathrm{mL})$ were strongly associated with mortality, which was independent of many risk factors, including age, sex, diabetes, previous myocardial infarction (MI), and high cholesterol levels [4]. In a nested casecontrol study of patients with previous MI, the risk of future MI increased progressively with increasing quartiles of baseline IL-6 concentration [5].

IL-6 is also predictive of cardiovascular events in patients with stable coronary disease. In a sub-study from the Stabilization of Atherosclerotic Plaque by Initiation of Darapladib Therapy Trial (STABILITY), higher levels of IL-6 were independently associated with the risk of major adverse cardiovascular events, cardiovascular and allcause mortality, MI, heart failure, and cancer mortality [6]. Recently, our group demonstrated an association between serum IL-6 concentrations and subclinical CAD, in which higher levels of serum IL-6 level (>1 pg/mL) were predictive of coronary stenosis $\geq 30 \%$ in intermediate-risk patients referred for coronary angiography [7]. 
Although there is a growing body of evidence associating IL-6 with

cardiovascular disease, most are indirect observations from case-control studies or subgroup analyses. IL-6 has been inadequately studied prospectively with respect to its predictive value. Much less is known about the role of IL-6 in patients referred for elective coronary angiography. Moreover, obesity and non-cardiovascular inflammatory diseases considerably interfere with serum IL-6 levels, which may lead to a confusing bias. In the present study, we aimed to prospectively analyze the impact of increased IL-6 levels on cardiovascular events in patients with high or very high cardiovascular risk, excluding severely obese patients and patients with previously known inflammatory conditions.

\section{METHODS}

\section{Study design}

This was an observational study divided into two phases: an initial crosssectional prospective phase and an observational cohort phase. The inclusion period was from October 2012 to August 2016. We screened potential participants who were referred to the cardiology division catheterization laboratory (Cath Lab) of Hospital de Clínicas, a large tertiary care university hospital in southern Brazil. We considered for inclusion every patient referred for elective coronary angiography due to non-acute chest pain or chronic myocardial ischemia confirmed by non-invasive investigation and who did not have any exclusion criteria (see below). Criteria were checked immediately before the procedure by the investigators by a hospital registry review and direct personal interview. If the patients qualified and agreed to participate in the study, a signed consent, anthropometric data, and a fasting blood sample for IL-6 and blood chemistry were obtained, and blood pressure was measured in the sitting position. 
After the procedure, patients were discharged from the unit and were referred to

102

103

104

105

106

107

108

109

110

111

112

113

114

115

116

117

118

119

their respective assistant physicians. From March 2020 to August 2020, all patients were contacted by one of the investigators through multiple phone calls and e-mail to obtain the most recent clinical information available, and their hospital and city obituary registries were reviewed.

\section{Inclusion and exclusion criteria}

We selected patients aged 30-80 years with suspected CAD due to a history of chronic chest pain or stable myocardial ischemia confirmed through a non-invasive test. We excluded patients with known class-IV New York Heart Association congestive heart failure, recent acute coronary syndrome (in the last 60 days), clinically significant renal disease (glomerular filtration rate $<45 \mathrm{~mL} / \mathrm{min} / 1.73 \mathrm{~m}^{2}$ ), any known inflammatory conditions such as chronic pulmonary obstructive disease, known chronic infectious diseases such as tuberculosis and HIV, rheumatic disease, chronic hepatitis B or C, thyroid disease, a history of organ transplantation or undergoing evaluation for transplantation, or known cancer. We also excluded severely obese patients with a body mass index $(\mathrm{BMI})>35 \mathrm{~kg} / \mathrm{m}^{2}$ and those taking medications, such as corticosteroids, HIVantiretroviral, carbamazepine, phenytoin, any drug for cancer, immunosuppressant, nitrofurantoin, anti-malaria, lithium, and anti-psychotic drugs, that might interfere with the inflammatory status of the patient. We did not exclude patients with diabetes or hypertension. Follow-up

All included patients were contacted through phone calls by one of the investigators from March to August 2020 and followed a specific protocol. Patients were 
required to confirm their clinical outcomes through medical registries. In-hospital registries were also obtained from those who continued to visit the hospital. Information regarding death was confirmed by a family member who attended the call, and their city obituary data were confirmed. Patients who could not be contacted after several attempts and had no further clinical hospital registry information after discharge were considered missing at random. contact if no outcomes occurred. made all the angiographic measurements. Angiographic analyses were made by visual 
projections. The presence of $\mathrm{CAD}$ was defined as any lesion causing $>30 \%$ reduction in the diameter of any epicardial coronary artery.

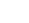

\author{
Clinical and biochemical investigation \\ Blood Pressure \\ Baseline blood pressure was measured at the Cath Lab after 15 min of rest, in
} the sitting position, in the right arm. Three sequential measurements were made using an automatic aneroid sphygmomanometer (OMRON Comfort III Visomat Incoterm, Germany). We considered the lowest blood pressure reading as the final measure.

IL-6 measures
Blood samples were collected at the Cath Lab just before the beginning of coronary angiography. For serum IL-6 measurement, a custom Luminex ${ }^{\circledR}$ assay was employed (Invitrogen ${ }^{\circledR}$, \#LHB0001CM) following the manufacturer's instructions. Briefly, $50 \mu \mathrm{L}$ of the undiluted sample was added to wells containing buffers and magnetic beads. After $2 \mathrm{~h}$ of incubation (550 rpm), the wells were washed and the detection antibody was added further for $1 \mathrm{~h}$. After washing, streptavidin-phycoerythrin was added further for $30 \mathrm{~min}$, the wells were washed again, and the beads were suspended in $125 \mu \mathrm{L}$ of wash buffer. Beads were read in Luminex ${ }^{\circledR} \mathrm{X}$-Map 200, and a minimum of 100 events were recorded for each bead. The limit of detection was defined as the lowest standard value $(0.08, \mathrm{pg} / \mathrm{mL})$. Values are expressed as $\mathrm{pg} / \mathrm{mL}$. Other assays Blood samples for high-sensitivity C-reactive protein (hs-CRP) were also collected simultaneously and aliquoted. Serum hs-CRP levels were determined using the turbidimetric immunoassay method (Roche®). Serum creatinine (Jaffé method), lipid 
profile, glycated hemoglobin (high performance liquid exchange chromatography), and glucose (colorimetric assay) were also measured.

\section{Statistical analysis}

Continuous variables were expressed as mean $( \pm$ standard deviation $[\mathrm{SD}])$ or median (interquartile range) based on their symmetrical or asymmetrical distribution, respectively. The normality of the distribution of each variable was assessed using the Shapiro-Wilk test. Categorical variables were represented by their relative and absolute frequencies.

Patients were divided according to their baseline IL-6 levels into 2 groups above and below $0.44 \mathrm{pg} / \mathrm{mL}$. This cut-off value was chosen from the ROC curve analysis as the best cut-off point for sensitivity and specificity. Patient groups were compared using the independent samples Student's t-test or Kruskal-Wallis test, as appropriate, for continuous variables and Fisher's exact tests for categorical variables. The Kaplan-Meier analyses and comparison using the log-rank test were performed using MedCalc Statistical Software version 14.8.1 (MedCalc Software bvba, Ostend, Belgium). All remaining statistical analyses were conducted using SPSS Statistics for Windows, Version 21.0. (IBM Corp., Armonk, NY, USA).

All patients provided written informed consent (the ethics committee of the Hospital de Clínicas approved the study protocol). 6

RESULTS

A flowchart of the inclusion process is depicted in Figure 1. A total of 4792 cardiac catheterizations were performed at the Cath Lab between October 2012 and 
August 2016. During this period, 141 patients were selected according to the inclusion and exclusion criteria. Of these, 100 were analyzed with complete follow-up data.

Baseline clinical and anthropometric characteristics are shown in Table 1. Fortyfour percent were men and the median age of all patients was 61.1 years. $\mathrm{CAD}$, that is > $30 \%$ stenosis was present in $81 \%$ of the patients and was similar between both sub-groups of IL-6. There was a higher proportion of patients with hypertension and type-2 diabetes (T2DM) and a trend toward a higher proportion of obese patients in the group with higher levels of IL-6. Hs-CRP levels, as expected, were also greater in the group with higher levels of IL-6.

The outcomes are shown in detail in Table 2. Overall, 32 cardiovascular events occurred during the follow-up period. The median overall time-to-event for the primary outcome was 297 weeks (95\% confidence interval $[\mathrm{CI}]=266.95-327.16)$. Figure 2 shows the Kaplan-Meier curves with the hazard ratios (HRs) of primary outcome between high and low IL-6 groups. There was a significantly higher cumulative incidence of the primary outcome during the follow-up period in the group of patients with increased baseline IL-6 $(\mathrm{HR}=2.81 ; 95 \% \mathrm{CI}=1.38 .5 .72, p$ for interaction $=0.015)$.

The area under the ROC curve of IL- 6 for the combined outcome was 0.585 $(95 \% \mathrm{CI}=0.482-0.683 ; p=0.156$; Figure 3) with a sensitivity of $81.25(95 \% \mathrm{CI}=$ 63.6-92.8), specificity of $38.24(95 \% \mathrm{CI}=26.7-50.8)$, positive predictive value of 38.2 $(95 \% \mathrm{CI}=26.7-50.8)$, and negative predictive value of $81.2(95 \% \mathrm{CI}=63.6-92.8)$.

A sub-group analysis (Figure 4) for the presence or absence of T2DM, hypertension, and obesity did not show any interaction among the subgroups with respect to the occurrence of outcomes. 
The present study shows that serum IL-6 is predictive of long-term

cardiovascular events in symptomatic patients with stable coronary disease who have a

high or very high cardiovascular risk. Serum IL-6 measurements $>0.44 \mathrm{pg} / \mathrm{mL}$ increased the risk of cardiovascular events by 2.8 times. Although there was an increased proportion of T2DM, hypertension, and obesity in the group with increased levels of IL-6, it was unlikely that it would have influenced the results, as the sub-group analysis showed no interaction among these sub-groups.

A previous study based on the analysis of two population-based cohorts [8] suggested that circulating serum IL-6 levels could be associated with increased coronary risk (defined as nonfatal MI or fatal coronary heart disease [CHD]). In that study, stored blood samples of patients who later developed non-fatal MI or died of CHD were used for baseline measurements. Patients who developed CHD had greater levels of IL-6 compared with controls with no history of CHD. The odds ratio for CHD, adjusted for several established risk factors, was $1.46(95 \% \mathrm{CI}=1.29-1.65)$ per 2 SDs of increase in baseline IL-6 values.

IL-6 has been associated with increased cardiovascular risk in some populations. In a meta-analysis of 29 population-based prospective studies [9], the adjusted relative risk for non-fatal MI or CHD death was 1.25 for every point of higher baseline SD in IL-

6. However, this meta-analysis had a considerable level of heterogeneity $\left(I^{2}=53.6 \%\right.$, $p=0.001$ ), and not all studies included indicated a clear risk prediction for IL-6. One possible reason is that many co-variables may have impacted the results in some studies. In the present study, we observed that the predictive cut-off value of IL-6 was relatively low $(0.44 \mathrm{pg} / \mathrm{mL})$ compared to that in other studies. In the sub-analysis of the STABILITY trial [6], the risk of cardiovascular death and major adverse cardiovascular 
events in 3-4 years started to increase progressively when IL-6 levels were above 1.5 ng/L. In the FRISC II trial [4], the highest predictive value of IL-6 was > 5 pg/L. We attribute our findings to the fact that we were able to exclude patients with chronic noncardiovascular inflammation, a great potential confounder when studying sub-clinical vascular inflammation. Moreover, we also had a low prevalence of obese patients in this population. It is known that there is a strong relationship ( $r h o=0.85 ; p<0.00001)$ between IL-6 levels and BMI [10]. We believe that the strict selection criteria we used improved the predictive value of IL-6, which may also explain the greater HRs for cardiovascular events in this study.

There is a clear plausibility for IL-6 levels to increase the risk of cardiovascular events. Experimental studies indicate that vascular endothelial and smooth muscle cells from normal and aneurysmal arteries can produce IL-6 [11,12]. Moreover, IL-6 gene transcripts are expressed in atherosclerotic lesions [13], confirming local production. IL6 has procoagulant effects [14], and elevated levels have been reported among patients with acute coronary syndromes [15]. Considering that atherosclerosis is a chronic inflammatory disorder, IL-6 levels are expected to be increased among individuals with sub-clinical atherosclerosis who are at greater risk for future MI. However, a cause-andeffect relationship between IL-6 and cardiovascular events cannot be clearly defined so far, as there is a lack of randomized trials targeting IL-6 treatment. However, in the Canakinumab Anti-inflammatory Thrombosis Outcome Study (CANTOS), a randomized, double-blind, placebo-controlled trial involving stable patients with previous MI, the human monoclonal antibody canakinumab, that targets the interleukin-1 $\beta$ innate immunity pathway, led to a significantly lower rate of recurrent cardiovascular events than the placebo, independent of lipid-level lowering [16], indicating a potential role for inflammatory mediators. 
276 of events. Thus, confirmation through larger studies is required. However, these results

277 should be considered preliminary, considering that statistical significance was achieved

278 in this small model. We also recognize that stricter adjustments are necessary to address

279 potential confounders, although the sub-group analysis certainly minimized this problem.

280

281

282

283

284

285

286

287

288

289

290

291

292

293

294

295

296

297

298

299

\section{CONCLUSION}

In conclusion, emergent markers of cardiovascular risk, such as IL-6, are still under evaluation. This preliminary prospective study indicates a role for IL-6 in reclassifying cardiovascular risk in high-risk patients, reinforcing the findings of some large retrospective studies and indicating the need for larger trials to evaluate the efficacy of lowering IL-6 in preventing cardiovascular events.

\section{List of Abbreviations:}

CAD: coronary artery disease

IL-6: interleukin-6

MI: myocardial infarction

Cath Lab: catheterization laboratory

T2DM: type-2 diabetes mellitus

CI: confidence interval

HR: hazard ratio

CHD: coronary heart disease

SD: standard deviation

ROC: Receiver operator characteristic 
DECLARATIONS

301 Ethics approval and consent to participate: All patients provided written informed consent, and the ethics committee of the Hospital de Clínicas approved the study protocol.

Competing interests: The authors have no conflicts of interest to declare and report no

304

305

306

307

308

309

310

311

312

313

314

315 financial relationships regarding the content.

Funding: Research Incentive Fund, Hospital de Clínicas de Porto Alegre (FIPE/HCPA). Availability of data and materials: The datasets during and/or analysed during the current study available from the corresponding author on reasonable request.

Consent for publication: not applicable

Authors' contributions: Stéfani Mariani, Márcio Mossmann, Gustavo Neves de Araújo and Sandro Cadaval Gonçalves recruited patients and collected data; Guilherme Pinheiro Machado analyzed statistical data; Marcello Bertoluci, Márcio Mossmann and Marco Wainstein wrote and review the manuscript. Marcello Bertoluci and Marco Wainstein were the mentors of the study.

Authorship declaration: All authors listed meet the authorship criteria according to the latest guidelines of the International Committee of Medical Journal Editors, and all authors are in agreement with the manuscript.

Acknowledgments: Not applicable.

\section{REFERENCES}

1. Heinrich PC, Castell JV, Andus T. Interleukin-6 and the acute phase response. Biochem J. 1990;265:621-36.

2. Solís-Herruzo JA, Rippe RA, Schrum LW, de la Torre P, García I, Jeffrey JJ et al. Interleukin-6 increases rat metalloproteinase-13 gene expression through stimulation of 
activator protein 1 transcription factor in cultured fibroblasts. $\mathrm{J}$ Biol Chem. $1999 ; 274: 30919-26$.

327 3. Galis ZS, Sukhova GK, Lark MW, Libby P. Increased expression of matrix metalloproteinases and matrix degrading activity in vulnerable regions of human atherosclerotic plaques. J Clin Invest. 1994;94:2493-503.

4. Lindmark E, Diderholm E, Wallentin L, Sigbahn A. Relationship between interleukin 6 and mortality in patients with unstable coronary artery disease: effects of an early invasive or noninvasive strategy. JAMA. 2001;286:2107-13.

5. Ridker PM, Rifai N, Stampfer M, Hennekens C. Plasma concentration of interleukin- 6 and the risk of future myocardial infarction among apparently healthy men. Circulation. 2000;101:1767-72.

6. Held C, White HD, Stewart RAH, Budaj A, Cannon CP, Hochman JS, et al. Inflammatory Biomarkers Interleukin. 6 and C-reactive protein and outcomes in stable coronary heart disease: experiences from the (stabilization of atherosclerotic plaque by initiation of darapladib therapy) STABILITY trial. J Am Heart Assoc. 2017 Oct 24;6(10):e005077.

7. Wainstein MV, Mossmann M, Araujo GN, Gonçalves SC, Gravina GL, Sangalli $M$ et al. Elevated serum interleukin-6 is predictive of coronary artery disease in intermediate risk overweight patients referred for coronary angiography. Diabetol Metab Syndr. 2017;9(1). 

term interleukin-6 levels and subsequent risk of coronary heart disease: two new prospective studies and a systematic review. PLOS Med. 2008;5:e78.

9. Kaptoge S, Seshasai SR, Gao P, Freitag DF, Butterworth AS, Borglykke A et al. Inflammatory cytokines and risk of coronary heart disease: new prospective study and updated meta-analysis. Eur Heart J. 2014;35:578-89.

357

10. Carey AL, Bruce CR, Sacchetti M, Anderson MJ, Olsen DB, Saltin B et al.

Interleukin-6 and tumor necrosis factor-alpha are not increased in patients with Type 2 diabetes: evidence that plasma interleukin-6 is related to fat mass and not insulin responsiveness. Diabetologia. 2004;47:1029-37.

362

11. Loppnow H, Libby P. Adult human vascular endothelial cells express the IL6 gene differentially in response to LPS or IL1. Cell Immunol. 1989;122:493-503.

12. Szekanecz Z, Shah MR, Pearce WH, Koch AE. Human atherosclerotic abdominal aortic aneurysms produce interleukin (IL)-6 and interferon-gamma but not IL-2 and ILActions. 1994;42:159-62.

13. Seino $Y$, Ikeda U, Ikeda M, Yamamoto $\mathrm{K}$, Misawa $\mathrm{Y}$, Hasegawa $\mathrm{T}$ et al. Interleukin-6 gene transcripts are expressed in human atherosclerotic lesions. Cytokine. 1994;6:87-91. 
375 14. Stouthard JML, Levi M, Hack CE, Beenhof CHN, Romijn HA, Sauerwein HP et 376 al. Interleukin-6 stimulates coagulation, not fibrinolysis, in humans. Thromb Haemost. 377 1996;76:738-42.

378

379 15. Biasucci LM, Liuzzo G, Fantuzzi G, Caligiuri G, Rebuzzi AG, Ginnetti F et al. 380 Increasing levels of interleukin (IL)-1Ra and IL-6 during the first 2 days of hospitalization 381 in unstable angina are associated with increased risk of in-hospital coronary events. 382 Circulation.1999;99:2079-84.

384 16. Ridker PM, Everett BM, Thuren T, MacFadyen JG, Chang WH, Ballantine C et al. CANTOS Trial Group. Anti-inflammatory therapy with canakinumab for atherosclerotic disease. N Engl J Med. 2017;377:1119-31.

FIGURE LEGENDS

390

391

Figure 1 - Flowchart of the inclusion process

392

Figure 2 - Time-to-Event Curves for composite outcome according IL-6 levels

393 Event rates are calculated with the use of Kaplan-Meier methods and compared with the 394 use of the log-rank test.

IL-6: interleukin-6

Figure 3 - Receiver operator characteristic (ROC) graph showing areas under the curve

IL-6: interleukin-6 
399 Figure 4 - Forrest plot of sub-group analysis for the presence or absence of T2DM, 400 hypertension, and obesity

401 T2DM: type-2 diabetes mellitus 
Figures

Screened $=4792$

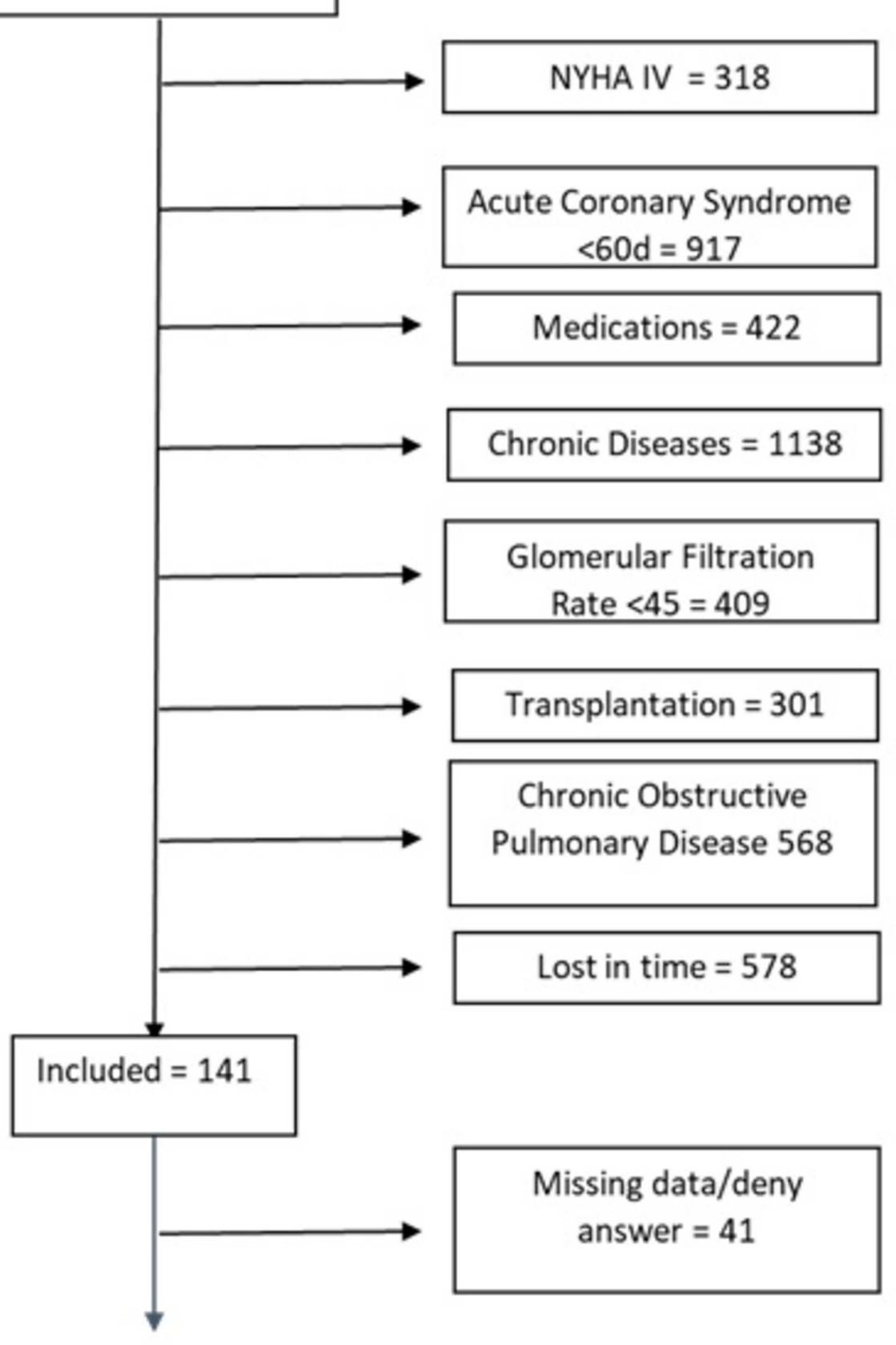

Analysed $=100$

phone call data $=44$

Clinical registries $=56$

Figure 1

Flowchart of the inclusion process 


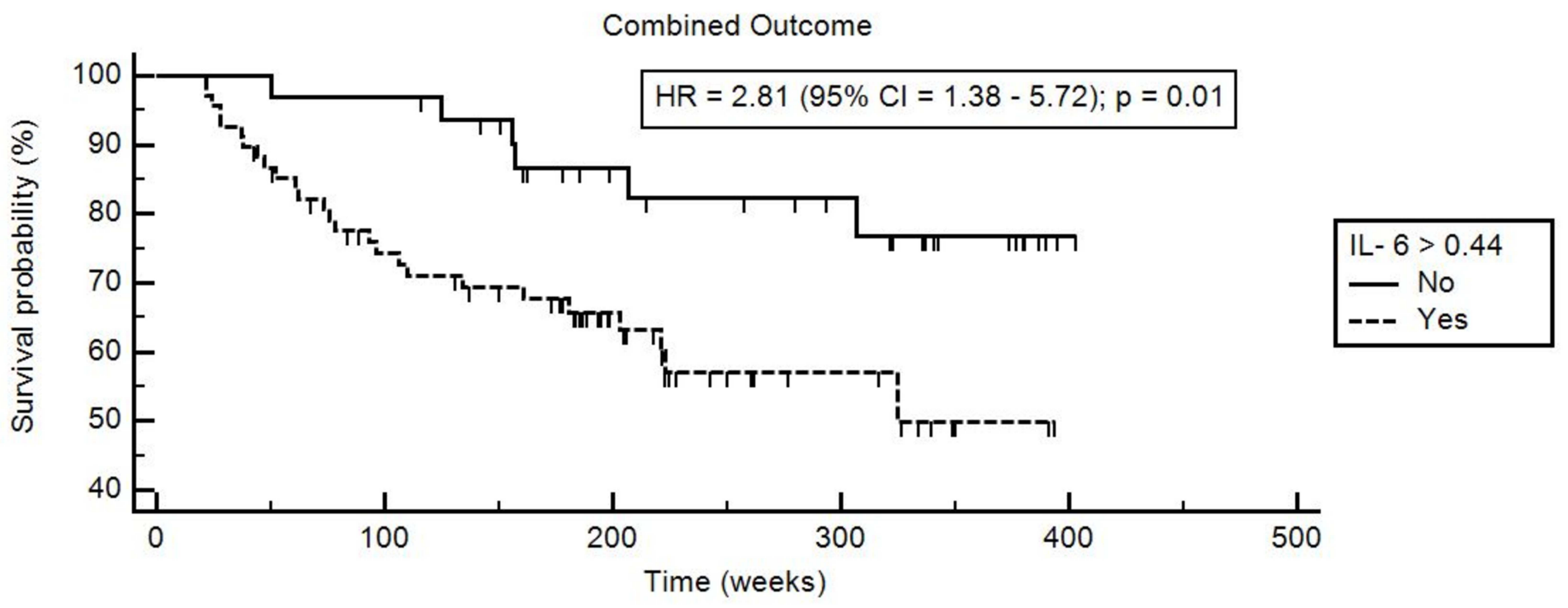

Number at risk

Group: No

$\begin{array}{rlcccc}32 & 31 & 20 & 15 & 1 & 0 \\ \text { Group: Yes } & & & & & \\ 68 & 46 & 25 & 9 & 0 & 0\end{array}$

Figure 2

Time-to-Event Curves for composite outcome according IL-6 levels Event rates are calculated with the use of Kaplan-Meier methods and compared with the use of the log-rank test. IL-6: interleukin-6 


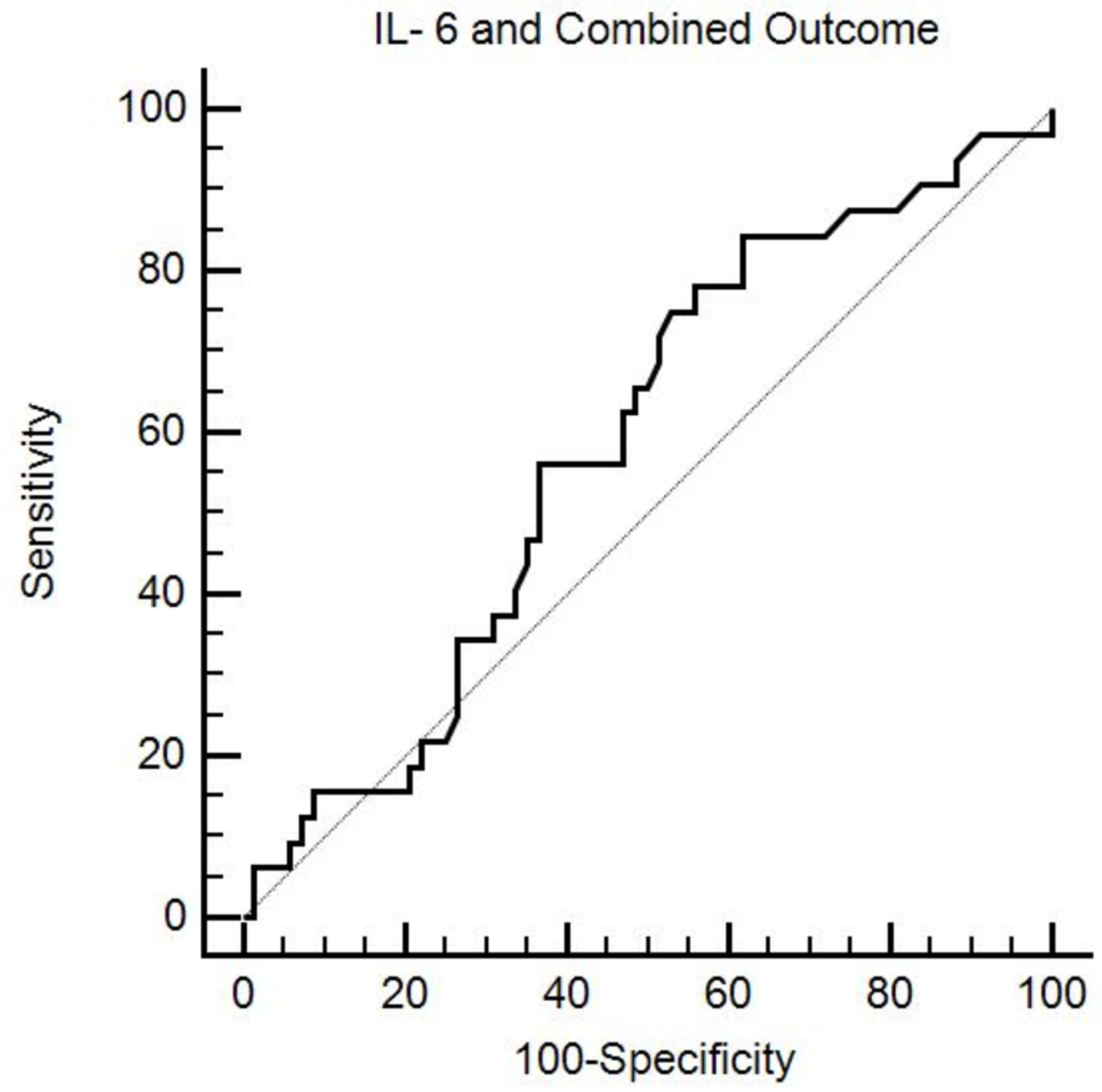

Figure 3

Receiver operator characteristic (ROC) graph showing areas under the curve IL- 6 for composite outcome IL-6: interleukin-6 


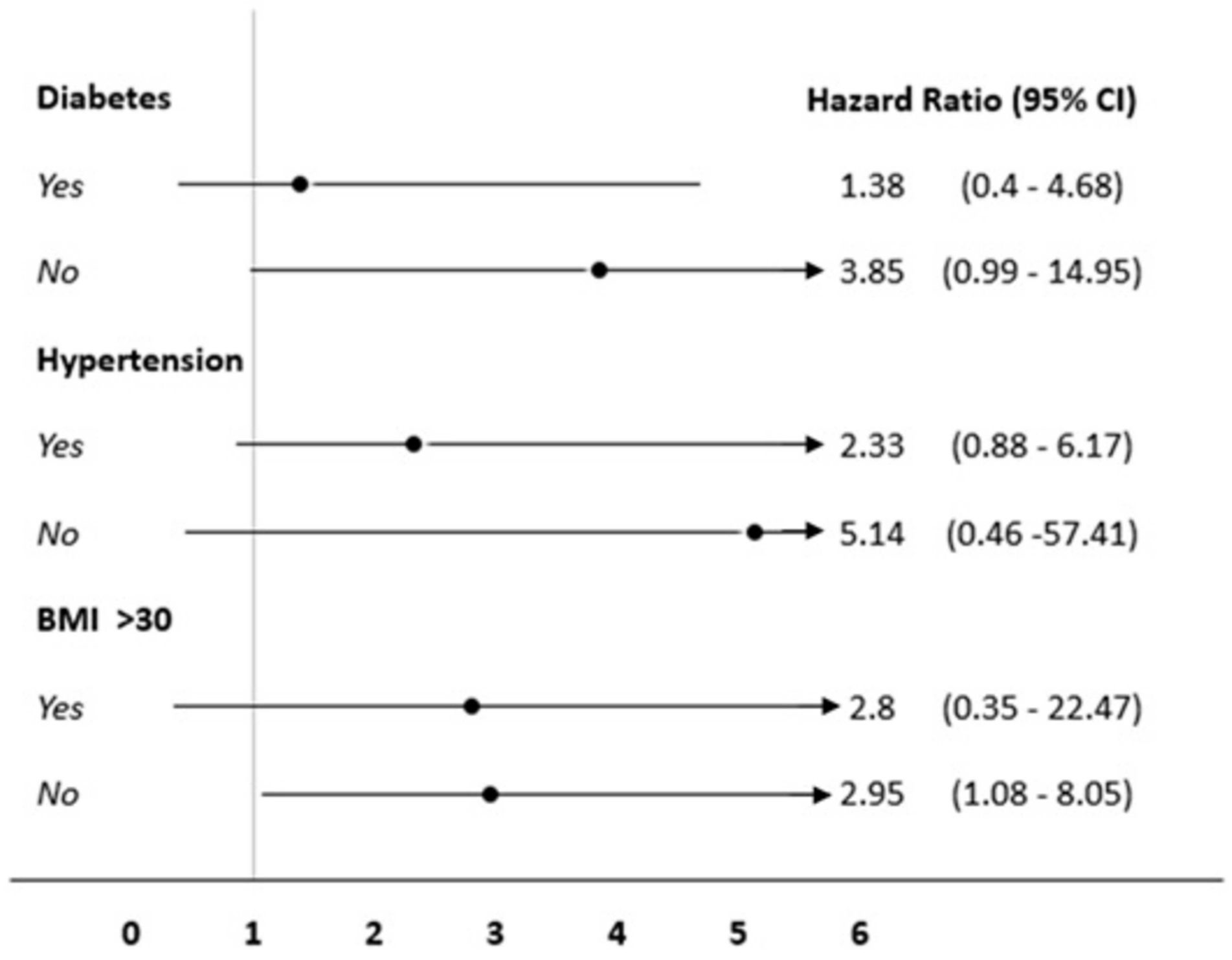

Figure 4

Forrest plot of sub-group analysis for the presence or absence of T2DM, hypertension, and obesityT2DM: type-2 diabetes mellitus 